\title{
MODELAGEM DA DISTRIBUIÇÃO DIAMÉTRICA DE ESPÉCIES FLORESTAIS EM UM FRAGMENTO DE FLORESTA OMBRÓFILA MISTA ${ }^{1}$
}

\author{
Enrique Orellana², Afonso Figueiredo Filho ${ }^{3}$, Sylvio Péllico Netto ${ }^{4}$ e Andrea Nogueira Dias ${ }^{3}$ \\ RESUMO - Este trabalho teve como objetivo avaliar o desempenho das funções densidade de probabilidade \\ (fdp) Weibull 3 Parâmetros (3P) e Beta ajustadas pelo método da Máxima Verossimilhança para descrever \\ a distribuição diamétrica de espécies arbóreas de um fragmento de Floresta Ombrófila Mista. A área de estudo \\ faz parte da Floresta Nacional de Irati (FLONA), onde foram instaladas 25 parcelas permanentes (100 m \\ x $100 \mathrm{~m}$ ) com área total amostrada de 25 ha e, para os ajustes, foram utilizados dados de todas as árvores \\ com diâmetro à altura do peito (DAP) superior ou igual a $10 \mathrm{~cm}$. Os ajustes foram feitos para as 20 espécies \\ com maior valor de importância (VI) com dados agrupados em classes de $5 \mathrm{~cm}$ de DAP. A programação não \\ linear (PNL) foi utilizada com o objetivo de minimizar os desvios entre valores observados e estimados. Para \\ avaliação dos ajustes, foram utilizados os testes de aderência de Kolmogorov-Smirnov e de Hollander-Proschan, \\ além do Erro-Padrão de Estimativa (\%) e Índice de Reynolds. Os resultados indicaram que as funções Weibull \\ 3P e Beta apresentaram resultados satisfatórios, que ambas poderiam ser recomendadas para avaliar a distribuição \\ diamétrica das espécies consideradas e que o uso da PNL nem sempre melhorou os ajustes.
}

Palavras-chave: Floresta de araucária; Funções densidade de probabilidade; Estrutura diamétrica.

\section{MODELING OF THE DIAMETER DISTRIBUTION FOR FOREST SPECIES IN A FRAGMENT OF MIXED OMBROPHYLLOUS FOREST}

\begin{abstract}
This study aimed to evaluate the performance of the probability density functions (pdf) Weibull with 3 parameters (3P) and Beta adjusted by Maximum Likelihood method to describe the diameter distribution of some representative species from a fragment of Araucaria Forest. The study area is part of the National Forest Irati (FLONA), where 25 permanent sample plots $(100 \mathrm{~m} \times 100 \mathrm{~m})$ were established with a total sampled area of $25 \mathrm{ha}$, and for the fittings, we used data from all trees with diameter at breast height (DBH) greater than or equal to $10 \mathrm{~cm}$. Adjustments were made for the 20 species with the highest importance value (VI) with data grouped into classes of $5 \mathrm{~cm} D B H$. The nonlinear programming (NLP) was used in order to minimize the deviations between observed and estimated values. To evaluate the adjustments, we used the Goodnessof-fit tests Kolmogorov-Smirnov and Hollander-Proschan, in addition to the Standard Error of the Estimate (\%) and Reynolds Index. The results indicated that Weibull 3P and Beta functions presented satisfactory results, and both could be recommended to evaluate the diameter distribution of the selected species and that the use of NLP has not improved adjustments in all cases.
\end{abstract}

Keywords: Araucaria forest; Density probability functions; Diametric structure.

\footnotetext{
${ }^{1}$ Recebido em 19.09.2011 aceito para publicação em 11.03.2014.

${ }^{2}$ Programa de Pós-Graduação em Ciências Florestais da Universidade Estadual do Centro-Oeste, UNICENTRO, Brasil. E-mail: <enriqueorellana@uol.com.br>.

${ }^{3}$ Universidade Estadual do Centro-Oeste, UNICENTRO, Brasil. E-mail: <afigfilho@gmail.com>e <andias@irati.unicentro.br>.

${ }^{4}$ Departamento de Ciências Florestais da Universidade Federal do Paraná, UFPR, Brasil. E-mail: <sylviopelliconetto@gmail.com>.
} 


\section{INTRODUÇÃO}

A Floresta Ombrófila Mista (FOM), ou Floresta com Araucária, é detentora de muitas espécies florestais endêmicas e de grande valor comercial. A extração de madeira de maneira insustentável e a expansão agrícola em décadas passadas, entre outros fatores, levaram essa tipologia florestal a uma expressiva redução em sua área de abrangência original.

Ainda que essa tipologia florestal esteja reduzida a fragmentos, é de fundamental importância que sejam desenvolvidos estudos voltados para o conhecimento da distribuição diamétrica de espécies analisadas individualmente, com o objetivo de avaliar a regeneração natural ou, mesmo, definir critérios de exploração, tendo em foco o manejo sustentável.

Segundo Bunyavejchewin et al. (2001), a distribuição diamétrica por espécie pode, frequentemente, prover informações adicionais a respeito das florestas, como distúrbios passados, principalmente se informações sobre tolerância à luz e padrões de estabelecimento da espécie são conhecidas. Loewenstein et al. (2000) citaram que, onde ocorrem espécies de crescimento lento e tolerantes à sombra, elas ocupam grande parte do estrato arbóreo inferior e, portanto, se apresentam em maior número na menor classe de diâmetro. Esses autores acrescentaram que, no entanto, espécies intolerantes à sombra (pioneiras) normalmente ocorrem com distribuição em forma de sino, característica de plantios equiâneos. Canalez et al. (2006) afirmaram que a forma de distribuição "J-invertido" de determinada espécie define que essa forma mantém a regeneração dentro de um fluxo contínuo. Entretanto, distribuições diamétricas com poucos elementos nas classes menores indicam descontinuidade de regeneração.

Machado et al. (1997) afirmaram que diversos são os modelos matemáticos apropriados para descrever distribuição de frequência por unidade de área em relação às classes sucessivas de diâmetro, e os mais conhecidos envolvem o uso de funções densidade probabilísticas.

Uma das funções densidade de probabilidades que mais se destacam no meio florestal é a função Weibull 3 Parâmetros (3P). Segundo Bailey e Dell (1973), se o parâmetro de forma $c<1$, a distribuição apresenta-se na forma de "J-invertido"; quando $c=1$, a distribuição é exponencial; e nos valores em que $1<c<3$, 6 a distribuição é unimodal, com assimetria positiva; quando $c=3,6$ é aproximadamente uma distribuição normal e, quando $c>3,6$ a distribuição tem assimetria negativa.

Outra função densidade de probabilidade que é bastante difundida em estudos de distribuições diamétricas em florestas é a função Beta. Finger (1992), citando-a, afirmou que ela pode gerar grande variedade de formas de distribuições decrescentes e unimodais, dependendo da magnitude dos expoentes.

O teste de aderência de Kolmogorov-Smirnov é bastante empregado na avaliação do desempenho de funções probabilísticas para descrever distribuições diamétricas. Esse teste compara a distribuição acumulada observada com a distribuição acumulada estimada pela função. Barra et al. (2004) propuseram uma metodologia para minimizar o maior desvio entre essas distribuições, utilizando um processo iterativo de otimização. Esses autores aplicaram essa metodologia ao testar o uso da programação não linear (PNL) na função Weibull 3P, comparando com o desempenho obtido pela função Weibull 2P sem o uso dessa ferramenta de otimização, para descrever a distribuição diamétrica de um povoamento de Populus sp.

O objetivo desta pesquisa foi avaliar o desempenho das funções Weibull 3P e Beta para descrever a distribuição diamétrica de 20 espécies de maior valor de importância em um fragmento de Floresta Ombrófila Mista.

\section{MATERIAL E MÉTODOS}

\section{1. Área de Estudo}

A Floresta Nacional (FLONA) de Irati é uma unidade de conservação localizada no Centro-Sul do Paraná, nos Municípios de Irati, Fernandes Pinheiro e Teixeira Soares, nas coordenadas geográficas de $25^{\circ} 25^{\prime}$ de latitude Sul, $50^{\circ} 36^{\prime}$ de longitude Oeste e $25^{\circ} 17^{\prime}$ de latitude Sul e $50^{\circ} 30^{\prime}$ de longitude Oeste (IBAMA, 2009).

O clima da região é “Cfb”, segundo a classificação de Köppen, com precipitação média anual de 1.442 mm e sem estação seca. A temperatura média em janeiro é de $22^{\circ} \mathrm{C}$, e a de julho fica em torno de $10^{\circ} \mathrm{C}$, com mais de cinco geadas por ano. O relevo local é considerado de ondulado a plano. O solo, classificado como Podzólico Vermelho-Amarelo, apresenta acidez média (DISPERATI et al., 2007). 
Em 2002, foram instaladas e medidas 25 parcelas permanentes com área de 1 ha (100 m x 100 m) cada uma, no fragmento de Floresta Ombrófila Mista da FLONA. Essas parcelas foram remedidas em 2005 e 2008, e nas três ocasiões todas as árvores com diâmetro à altura do peito (DAP) igual ou superior a $10 \mathrm{~cm}$ foram medidas e identificadas.

\subsection{Ajuste e avaliação das funções-densidade de probabilidade}

Foram ajustadas as funções densidade de probabilidade Beta e Weibull 3 parâmetros (3P) pelo método da Máxima Verossimilhança, com e sem o uso da Programação Não Linear (PNL).

\subsubsection{Função Weibull $3 P$}

Segundo Bailey e Dell (1973) e Campos e Turnbull (1981), a função densidade de probabilidade da Weibull 3 parâmetros $f(x)$ e respectiva distribuição acumulativa $\mathrm{F}(\mathrm{x})$ são expressas pelas fórmulas:

$$
\begin{aligned}
& \mathrm{f}(\mathrm{x})=\frac{\mathrm{c}}{\mathrm{b}}\left(\frac{\mathrm{x}-\mathrm{a}}{\mathrm{b}}\right)^{\mathrm{c}-1} \exp \left[-\left(\frac{\mathrm{x}-\mathrm{a}}{\mathrm{b}}\right)^{\mathrm{c}}\right] \\
& \mathrm{F}(\mathrm{x})=1-\exp \left[-\left(\frac{\mathrm{x}-\mathrm{a}}{\mathrm{b}}\right)^{\mathrm{c}}\right] \\
& \text { em que: } \\
& \mathrm{a}=\text { parâmetro de localização; } \\
& \mathrm{b}=\text { parâmetro de escala; e } \\
& \mathrm{c}=\text { parâmetro de forma. }
\end{aligned}
$$

\subsubsection{Função Beta}

Segundo Scolforo (1998), a função Beta pode ser descrita como:

$f(x)=\frac{\Gamma(\alpha+\beta)}{\Gamma(\alpha) \Gamma(\beta)} \cdot \frac{1}{\left(d_{\max }-d_{\min }\right)^{\alpha+\beta-1}} \cdot\left(d-d_{\min }\right)^{\alpha-1}\left(d_{\max }-d\right)^{\beta-1}$

$\left\{\begin{array}{c}\mathrm{d}_{\min } \leq \mathrm{x} \leq \mathrm{d}_{\max } \\ 0 \text { em outras ocasiões }\end{array}\right.$

$\Gamma$ é a função Gama, e os parâmetros $\alpha$ e $\beta$ definem a forma da distribuição.

\subsection{Programação Não Linear (PNL) para ajustes de funções}

A Programação Não Linear (PNL) é ferramenta de otimização da Pesquisa Operacional pertencente à Programação Matemática. A PNL foi utilizada em ambas as funções testadas, conforme metodologia proposta por Barra et al. (2004) para a função Weibull 3P.

Esses autores recomendaram a utilização da Programação Não Linear como ferramenta para minimizar a maior diferença $\left(d_{n}\right)$ entre a frequência observada acumulada e a frequência estimada acumulada, positiva $\left(d_{n}^{+}\right)$ou negativa $\left(d_{n}^{-}\right)$, utilizada no teste de KolmogorovSmirnov. Desse modo, a função objetivo apresenta a seguinte forma:

$$
\operatorname{Min} \mathrm{d}_{\mathrm{n}}=\operatorname{Min}\left\{\operatorname{Max}\left\{\mathrm{d}_{\mathrm{n}}^{+}\left|\mathrm{d}_{\mathrm{n}}^{-}\right|\right\}\right\}
$$

Na PNL, as restrições para a função Weibull 3P empregadas no trabalho foram:

$$
\begin{aligned}
& \mathrm{a} \geq 0 \\
& \mathrm{a}<\mathrm{DAP}_{\text {min }} \\
& \mathrm{b}>0 \\
& \mathrm{c}>0
\end{aligned}
$$

O ajuste foi realizado com o software SOLVER, que utiliza o algoritmo linear Gradiente Reduzido Generalizado (GRG2) para aperfeiçoar problemas não lineares. Neste trabalho, foi utilizada também a Programação Não Linear para a função Beta. Nesse caso, as restrições utilizadas foram de que $\alpha \geq 0$ e $\beta \geq 0$.

\subsection{Estatísticas utilizadas para avaliar o desempenho das funções densidade de probabilidade}

A análise do desempenho das funções ajustadas foi feita a partir dos testes de aderência de KolmogorovSmirnov (K-S) e de Hollander-Proschan (H-S), análise dos resíduos, Erro-Padrão da Estimativa (EPE\%) e o índice de erro proposto por Reynolds et al. (1988).

\subsubsection{Teste de Kolmogorov-Smirnov (K-S)}

$$
D_{\text {calc }}=\sup |\operatorname{Fo}(x)-F e(x)|
$$

$$
\text { em que: }
$$

Fo(x) $=$ proporção dos valores de frequência observada acumulada;

Revista Árvore, Viçosa-MG, v.38, n.2, p.297-308, 2014 
$\mathrm{Fe}(\mathrm{x})$ = proporção dos valores de frequência esperada acumulada; e

$\mathrm{D}_{\text {calc }}=$ módulo do desvio máximo observado.

O nível de probabilidade usado para $\mathrm{N}$ indivíduos foi $\alpha=0,01$.

\subsubsection{Teste de Hollander-Proschan (H-P)}

Segundo Dodson (2006), o teste compara a função de sobrevivência teórica ajustada $\mathrm{S}_{0}(\mathrm{t})$ com a estimativa de Kaplan-Meier $S(t)$ para a função de sobrevivência subjacente $S(t)$, com hipótese nula dada por:

$$
\mathrm{H}_{0}: \mathrm{S}(\mathrm{t})=\mathrm{S}_{0}(\mathrm{t})
$$

A função de sobrevivência teórica é obtida como:

$$
\mathrm{S}_{0}(\mathrm{t})=1-\mathrm{F}(\mathrm{t})
$$

A estatística para o teste de Hollander e Proschan para a hipótese nula de que os dados são provenientes da distribuição com função de sobrevivência $S_{0}(x)$ é dada por:

$$
C=\sum_{i=1}^{n} S_{0}\left(t_{i}\right) \hat{f}\left(t_{i}\right)
$$

Sob a hipótese nula, a estatística:

$$
\mathrm{C}^{*}=\frac{\sqrt{\mathrm{n}}(\mathrm{C}-0,5)}{\hat{\sigma}}
$$

segue aproximadamente a distribuição normal padronizada, em que $\hat{\sigma}$ é uma estimativa do desviopadrão de C:

$$
\hat{\sigma}^{2}=\frac{1}{16} \sum_{i=1}^{n} \frac{n}{n-i+1}\left[S_{0}^{2}\left(t_{i-1}\right)-S_{0}^{2}\left(t_{i}\right)\right]
$$

Os valores de $\mathrm{S}_{0}(\mathrm{t})$ para observações repetidas se mantêm constantes, o que permite o cálculo de $\hat{\sigma}^{2}$ a partir das observações distintas, sem considerar as frequências.

Rejeita-se $\mathrm{H}_{0}$ : $\mathrm{S}(\mathrm{t})=\mathrm{S}_{0}(\mathrm{t})$ se $\left|\mathrm{C}^{*}\right|>\mathrm{Z}_{\alpha / 2}$, em nível $\alpha$ de significância. Neste trabalho, o nível de significância foi de $95 \%$. Assim, o $Z$ tabelado foi de 1,96. Portanto, se $-1,96<C^{*}<1,96$, existe aderência da função com a distribuição analisada.

\subsection{3. Índice de Reynolds}

Alguns autores (TORRES-ROJO et al., 2000; CAO, 2004; QIN et al., 2007; PEUHKURINEN et al., 2008;
SIIPILEHTO, 2009) têm utilizado o índice de Reynolds para avaliar funções densidade de probabilidade para caracterizar distribuições diamétricas.

Segundo Qin et al. (2007), o índice proposto por Reynolds et al. (1988) determina o desempenho dos métodos por classe diamétrica. Torres-Rojo et al. (2000) citaram que o índice de Reynolds é definido como a soma ponderada das diferenças absolutas entre o número de árvores observado e estimado das árvores em cada classe diamétrica. Esses autores afirmaram ainda que a ponderação pode ser feita com o volume ou valor da madeira, apesar de que Reynolds et al. (1988) tenham afirmado que outra ponderação pode ser utilizada. TorresRojo et al. (2000) e Peuhkurinen et al. (2008) utilizaram a área basal observada em cada classe diamétrica para fins de ponderação.

Podlaski (2006) comparou o índice de Reynolds com e sem a ponderação da área basal para avaliar distribuições diamétricas. Concluiu que sua utilização para fins de ponderação não apresentou efeitos significativos na análise das distribuições.

Siipilehto (2009); Cao (2004) e Qin et al. (2007) não utilizaram ponderação. Eles consideraram apenas o somatório dos desvios absolutos entre valores observados e estimados nas classes diamétricas, para o cálculo do índice de Reynolds:

$$
\mathrm{IR}=\sum_{\mathrm{i}=1}^{\mathrm{k}}\left|\mathrm{N}_{\mathrm{i}}-\hat{\mathrm{N}}_{\mathrm{i}}\right|
$$

em que:

IR= Índice de Reynolds;

$\mathrm{N}_{\mathrm{i}}=$ número de árvores por hectare observado na classe $i$; e

$\mathrm{N}_{\mathrm{i}}=$ número de árvores por hectare estimado na classe $i$.

Segundo Qin et al. (2007), um baixo valor de IR indica pequena diferença entre o número de árvores observado e o estimado nas classes de diâmetro.

\subsubsection{Erro-Padrão de Estimativa (EPE)}

O Erro-Padrão de Estimativa (EPE) foi calculado pela fórmula normalmente aplicada para avaliar a regressão em modelos matemáticos, sendo:

$$
\mathrm{EPE} \%=\frac{\mathrm{EPE}}{\overline{\mathrm{Y}}} \cdot 100
$$


A média $(\bar{Y})$ foi calculada pela fórmula para dados agrupados em classes.

$$
E P E=\sqrt{\frac{\sum_{i=1}^{n}\left(Y_{i}-\hat{Y}_{i}\right)^{2}}{n-p}}
$$

em que :

$$
\begin{aligned}
& \text { EPE = Erro-Padrão de Estimativa; } \\
& \mathrm{N}=\text { número total de árvores por hectare; } \\
& \mathrm{Y}_{\mathrm{i}}=\mathrm{n}^{\circ} \text { de árvores por hectare observado na classe } i \text {; } \\
& \hat{\mathrm{Y}}_{\mathrm{i}}=\mathrm{n}^{\mathrm{o}} \text { de árvores por hectare estimado na classe } i \text {; e } \\
& \mathrm{P}=\text { número de parâmetros da função. }
\end{aligned}
$$

\subsection{Tratamento dos dados}

A partir da análise fitossociológica realizada por Sawczuk (2009), com os dados medidos no ano 2008, foram selecionadas as 20 espécies com maior valor de importância (VI) para a modelagem da distribuição diamétrica, colocadas em ordem decrescente de importância: Araucaria angustifolia (araucária), Ilex paraguariensis (erva-mate), Ocotea odorifera (canelasassafrás), Nectandra grandiflora (canela-amarela), Ocotea porosa (imbuia), Casearia decandra (guaçatunga-branca), Cedrela fissilis (cedro), Ocotea puberula (canela-guaicá), Matayba elaeagnoides (miguel-pintado), Nectandra megapotamica (canelaimbuia), Coussarea contracta (cinzeiro-preto), Myrsine umbellata (capororocão), Capsicodendron dinisii (pimenteira), Ocotea diospyrifolia (canela-pimenta), Casearia sylvestris (guaçatunga-preta), Prunus brasiliensis (pessegueiro-bravo), Ilex theezans (caúna), Myrcia hebepetala (caingá), Dalbergia brasiliensis (jacarandá) e Campomanesia xanthocarpa (guabiroba).

Para os ajustes, foram utilizados todos os diâmetros (DAP) das árvores de cada espécie selecionada na área amostrada (25 ha) para dados agrupados em classes com amplitude de $5 \mathrm{~cm}$ de diâmetro. Em seguida, foi aplicada a PNL nos dois ajustes realizados em cada espécie (um para cada função testada), com o objetivo de melhorar as estimativas entre valores observados e estimados. O método de ajuste com o menor $\mathrm{D}_{\text {calc }}$ do teste de Kolmogorov-Smirnov foi considerado o que apresentou o melhor desempenho entre os quatro analisados (com e sem o uso da PNL).

\section{RESULTADOS}

A Tabela 1 apresenta as principais estatísticas por espécie em ordem de maior VI da área de estudo.

A Tabela 2 apresenta os coeficientes e estatísticas dos ajustes das funções consideradas de melhor desempenho das espécies selecionadas.

As estatísticas da Tabela 2 indicam que houve aderência dos valores estimados pelas funções Weibull e Beta com a frequência observada no teste de Kolmogorov-Smirnov, em todos os ajustes realizados. Para o teste de Hollander-Proschan, não houve aderência de apenas quatro espécies.

Considerando o índice de Reynolds, entre as 20 espécies analisadas, em 10 delas a função Weibull 3P foi melhor e, em outras 10, a função Beta apresentou melhores estimativas. Das 20 espécies analisadas, a Weibull 3P sem PNL teve melhor desempenho em oito vezes (40\%), a Weibull 3P com PNL em duas vezes (10\%), a Beta sem PNL em quatro vezes (20\%) e a Beta com PNL em seis vezes (30\%).

Ao avaliar o EPE (\%) das 20 espécies, a Weibull 3P apresentou as melhores estimativas em 11 ajustes realizados, enquanto a função Beta foi melhor em nove deles. Entre os 20 ajustes, a Weibull 3P sem PNL apresentou melhor desempenho em 10 vezes (50\%), a Weibull 3P com PNL em uma vez (5\%), a Beta sem PNL em cinco vezes (25\%) e a Beta com PNL em quatro vezes (20\%). Houve disparidade entre as estatísticas Índice de Reynolds e EPE (\%) em apenas cinco casos (araucária, imbuia, cedro, pimenteira e guabiroba).

Com os coeficientes apresentados na Tabela 2, foram geradas as estimativas do número de árvores por hectare em cada classe (Figuras 1).

O parâmetro $c$ da função Weibull 3P apresentou valores acima de 1 em algumas espécies, como é o caso da Ilex paraguariensis, Casearia decandra, Myrsine umbellata, Prunus brasiliensis e Ilex theezans, o que caracteriza as distribuições unimodais com assimetria positiva. Porém, analisando-se as Figuras 1, essas espécies apresentaram a forma decrescente, mesmo quando o parâmetro de forma $c$ apresentou valores acima de 1. Uma justificativa para a ocorrência dessa inconsistência é que o intervalo de $5 \mathrm{~cm}$ utilizado para agrupar os dados de algumas espécies aparentemente não representou a realidade da distribuição, principalmente nas primeiras classes, nas quais o número

Revista Árvore, Viçosa-MG, v.38, n.2, p.297-308, 2014 
Tabela 1 - Espécies de maior valor de importância (VI) e principais estatísticas do diâmetro à altura do peito - DAP (cm). Table 1 -Species with the highest importance values (VI) and the main statistics of diameter at breast height- DBH (cm).

\begin{tabular}{|c|c|c|c|c|c|}
\hline Espécies & No de árvores* & N/ha & Média & Mediana & Máx. \\
\hline Araucaria angustifolia (araucária) & 1058 & 42 & 43,63 & 43,29 & 121,44 \\
\hline Ilex paraguariensis (erva-mate) & 1334 & 53 & 18,20 & 17,19 & 43,77 \\
\hline Ocotea odorifera (canela-sassafrás) & 1315 & 53 & 18,82 & 17,63 & 56,34 \\
\hline Nectandra grandiflora (canela-amarela) & 885 & 35 & 24,12 & 22,60 & 65,25 \\
\hline Ocotea porosa (imbuia) & 480 & 19 & 38,65 & 36,64 & 155,97 \\
\hline Casearia decandra (guaçatunga-branca) & 840 & 34 & 16,35 & 15,06 & 45,96 \\
\hline Cedrela fissilis (cedro) & 378 & 15 & 32,30 & 31,42 & 82,60 \\
\hline Ocotea puberula (canela-guaicá) & 329 & 13 & 34,21 & 31,83 & 86,58 \\
\hline Matayba elaeagnoides (miguel-pintado) & 395 & 16 & 28,24 & 27,37 & 61,02 \\
\hline Nectandra megapotamica (canela-imbuia) & 443 & 18 & 24,10 & 22,12 & 73,53 \\
\hline Coussarea contracta (cinzeiro-preto) & 626 & 25 & 13,13 & 12,41 & 30,56 \\
\hline Myrsine umbellata (capororocão) & 486 & 19 & 16,24 & 15,28 & 35,97 \\
\hline Capsicodendron dinisii (pimenteira) & 317 & 13 & 23,41 & 21,87 & 55,70 \\
\hline Ocotea diospyrifolia (canela-pimenta) & 274 & 11 & 28,37 & 28,20 & 69,07 \\
\hline Casearia sylvestris (guaçatunga-preta) & 356 & 14 & 16,22 & 15,36 & 34,06 \\
\hline Prunus brasiliensis (pessegueiro-bravo) & 273 & 11 & 17,25 & 15,44 & 43,45 \\
\hline Ilex theezans (caúna) & 285 & 11 & 15,88 & 14,80 & 40,11 \\
\hline Myrcia hebepetala (caingá) & 284 & 11 & 13,49 & 12,73 & 31,26 \\
\hline Dalbergia brasiliensis (jacarandá) & 236 & 9 & 18,40 & 17,35 & 38,04 \\
\hline Campomanesia xanthocarpa (guabiroba) & 162 & 6 & 23,80 & 20,69 & 49,66 \\
\hline
\end{tabular}

*Número total de árvores utilizadas no ajuste, considerando-se toda a área amostrada (25 ha).

de árvores é maior. Sabe-se que, quanto menor o intervalo de classe utilizado, maior a visualização real da distribuição. Segundo Westphal et al. (2006), intervalos de classes maiores podem atenuar as pequenas irregularidades na distribuição.

Para exemplificar o exposto, os dados de diâmetro da Ilex paraguariensis e da Myrsine umbellata foram agrupados em intervalos de $2 \mathrm{~cm}$. Verificou-se que a distribuição passou de decrescente, quando os dados foram agrupados em $5 \mathrm{~cm}$ (Figuras 1), para unimodal com forte assimetria positiva, conforme demonstrado na Figura 2.

\section{DISCUSSÃO}

Os resultados gerais indicam que ambas as funções (Weibull 3P e Beta) podem ser utilizadas para as espécies analisadas, visto que houve aderência do teste K-S em todos os casos, ou seja, não houve diferença significativa entre a distribuição real e a distribuição estimada.

A utilização da Programação Não Linear (PNL) nem sempre melhorou os ajustes, conforme se esperava. Embora sua aplicação tenha visado reduzir a maior diferença no teste K-S entre as distribuições observada e estimada com dados agrupados em classes, a Tabela 2 indica que a ferramenta de otimização diminuiu o desvio em oito das 20 espécies florestais avaliadas. Nos casos em que houve contribuição na melhoria dos ajustes com o uso dessa ferramenta, a distribuição apresentou tendência decrescente e, em outros casos, tendência unimodal, indicando que é ferramenta versátil e que pode promover a melhoria de funções probabilísticas que se ajustem às diversas formas de distribuições diamétricas de espécies florestais encontradas em florestas nativas.

Nos casos em que a PNL não contribuiu com a melhoria dos ajustes, a ferramenta de otimização de fato diminuiu a diferença na classe de maior desvio, no entanto resultou em maior desvio em outro ponto da distribuição, não contribuindo, portanto, para a melhoria dos ajustes nesses casos. O resultado do trabalho de Barra et al. (2004) indicou melhoria no ajuste com o uso da otimização para descrever a distribuição diamétrica unimodal de um povoamento de Populus sp.

Barros (1980), em estudos realizados na floresta do Planalto Tapajós, na Amazônia, testou várias funções densidade de probabilidade, entre elas a Exponencial (Meyer tipo I e II), Weibull 2 parâmetros, Polinomial de Goff \& West, e Beta, para ajustar a distribuição diamétrica de todas as espécies, algumas de valor 
Tabela 2 - Coeficientes e estatísticas das funções/métodos ajustados com maior desempenho das 20 espécies de maior valor de importância (VI).

Table 2 - Coefficients and statistics of the functions/methods adjusted with best performance for the 20 species with the highest importance value (VI).

\begin{tabular}{|c|c|c|c|c|c|}
\hline Espécies & Função & Coeficientes & $\mathrm{D}_{\text {calc }} / \mathrm{C}^{*}$ & IR & $\operatorname{EPE}(\%)$ \\
\hline Araucaria angustifolia & BETA COM PNL & $\begin{array}{l}\alpha=1,347 \\
\beta=3,033\end{array}$ & $0,0361^{1 / 0}, 18^{2}$ & 9,25 & 0,98 \\
\hline Ilex paraguariensis & WEIBULL COM PNL & $\begin{array}{l}\mathrm{A}=10,02 \\
\mathrm{~B}=9,489 \\
\mathrm{C}=1,352\end{array}$ & $0,0251^{1 / 1}, 96^{2}$ & 2,52 & 0,86 \\
\hline Ocotea odorifera & BETA COM PNL & $\begin{array}{l}\alpha=1,452 \\
\beta=6,282\end{array}$ & $0,0084^{1 /-0,17}$ & 3,12 & 1,01 \\
\hline Nectandra grandiflora & WEIBULL SEM PNL & $\begin{array}{l}\mathrm{A}=10,02 \\
\mathrm{~B}=15,53 \\
\mathrm{C}=1,450\end{array}$ & $0,0292^{1 /-0,20}$ & 2,66 & 0,85 \\
\hline Ocotea porosa & BETA COM PNL & $\begin{array}{l}\alpha=1,809 \\
\beta=7,422\end{array}$ & $0,0287^{1 /}-0,16^{2}$ & 3,88 & 0,88 \\
\hline Casearia decandra & WEIBULL SEM PNL & $\begin{array}{l}\mathrm{A}=10,02 \\
\mathrm{~B}=6,695 \\
\mathrm{C}=1,178\end{array}$ & $0,0288^{1 /-0,06}$ & 2,20 & 0,97 \\
\hline Cedrela fissilis & WEIBULL SEM PNL & $\begin{array}{l}\mathrm{A}=10,02 \\
\mathrm{~B}=24,62 \\
\mathrm{C}=1,578\end{array}$ & $0,0739^{1 /-0,93^{2}}$ & 2,64 & 0,95 \\
\hline Ocotea puberula & WEIBULL SEM PNL & $\begin{array}{l}\mathrm{A}=10,02 \\
\mathrm{~B}=26,87 \\
\mathrm{C}=1,577\end{array}$ & $0,0851^{1 /-0,29^{2}}$ & 2,41 & 0,63 \\
\hline Matayba elaeagnoides & BETA SEM PNL & $\begin{array}{l}\alpha=1,304 \\
\beta=2,351\end{array}$ & $0,0364^{1 /-0,16^{2}}$ & 2,04 & 0,85 \\
\hline Nectandra megapotamica & BETA COM PNL & $\begin{array}{l}\alpha=0,988 \\
\beta=3,463\end{array}$ & $0,0163^{1 / 0,14^{2}}$ & 2,28 & 0,94 \\
\hline Coussarea contracta & BETA COM PNL & $\begin{array}{l}\alpha=0,827 \\
\beta=5,062\end{array}$ & $0,0016^{1 / 1}, 04^{2}$ & 0,13 & 0,10 \\
\hline Myrsine umbellata & WEIBULL SEM PNL & $\begin{aligned} \mathrm{A} & =10,02 \\
\mathrm{~B} & =6,738 \\
\mathrm{C} & =1,32\end{aligned}$ & $0,0363^{1 /-0,41^{2}}$ & 1,07 & 0,79 \\
\hline Capsicodendron dinisii & BETA SEM PNL & $\begin{array}{l}\alpha=1,127 \\
\beta=2,615\end{array}$ & $0,0254^{1} / 0,28^{2}$ & 1,38 & 0,73 \\
\hline Ocotea diospyrifolia & BETA SEM PNL & $\begin{array}{l}\alpha=0,890 \\
\beta=2,000\end{array}$ & $0,0672^{1 /-0,55^{2}}$ & 2,52 & 1,26 \\
\hline Casearia sylvestris & BETA SEM PNL & $\begin{array}{l}\alpha=0,953 \\
\beta=2,664\end{array}$ & $0,0245^{1 / 0}, 27^{2}$ & 1,09 & 1,01 \\
\hline Prunus brasiliensis & WEIBULL SEM PNL & $\begin{array}{l}\mathrm{A}=10,02 \\
\mathrm{~B}=7,503 \\
\mathrm{C}=1,108\end{array}$ & $0,0808^{1 /-0,12^{2}}$ & 1,15 & 0,75 \\
\hline Ilex theezans & WEIBULL SEM PNL & $\begin{array}{l}\mathrm{A}=10,02 \\
\mathrm{~B}=6,248 \\
\mathrm{C}=1,160\end{array}$ & $0,0615^{1 / 0,80^{2}}$ & 1,02 & 0,84 \\
\hline Myrcia hebepetala & BETA COM PNL & $\begin{array}{l}\alpha=0,836 \\
\beta=4,670\end{array}$ & $0,0070^{1 /-2,22}$ & 0,32 & 0,44 \\
\hline Dalbergia brasiliensis & WEIBULL SEM PNL & $\begin{array}{l}\mathrm{A}=10,02 \\
\mathrm{~B}=9,280 \\
\mathrm{C}=1,550\end{array}$ & $0,0748^{1 /-0,36^{2}}$ & 1,14 & 0,84 \\
\hline \multicolumn{2}{|c|}{ Campomanesia xanthocarpa WEIBULL COM PNL } & $\begin{array}{l}\mathrm{A}=10,02 \\
\mathrm{~B}=17,90 \\
\mathrm{C}=0,910\end{array}$ & $0,1252^{1 / 1}, 60^{2}$ & 1,06 & 1,16 \\
\hline
\end{tabular}

${ }^{1}$ Houve aderência em nível de 0,01 de probabilidade pelo teste K-S; ${ }^{2}$ Houve aderência em nível de 0,05 de probabilidade pelo teste HP; PNL - programação não linear.; $\mathrm{D}_{\text {calc }}=$ estatística do teste K-S, C* = estatística do teste H-P; IR = Índice de Reynolds; e EPE = erro-padrão da estimativa. 

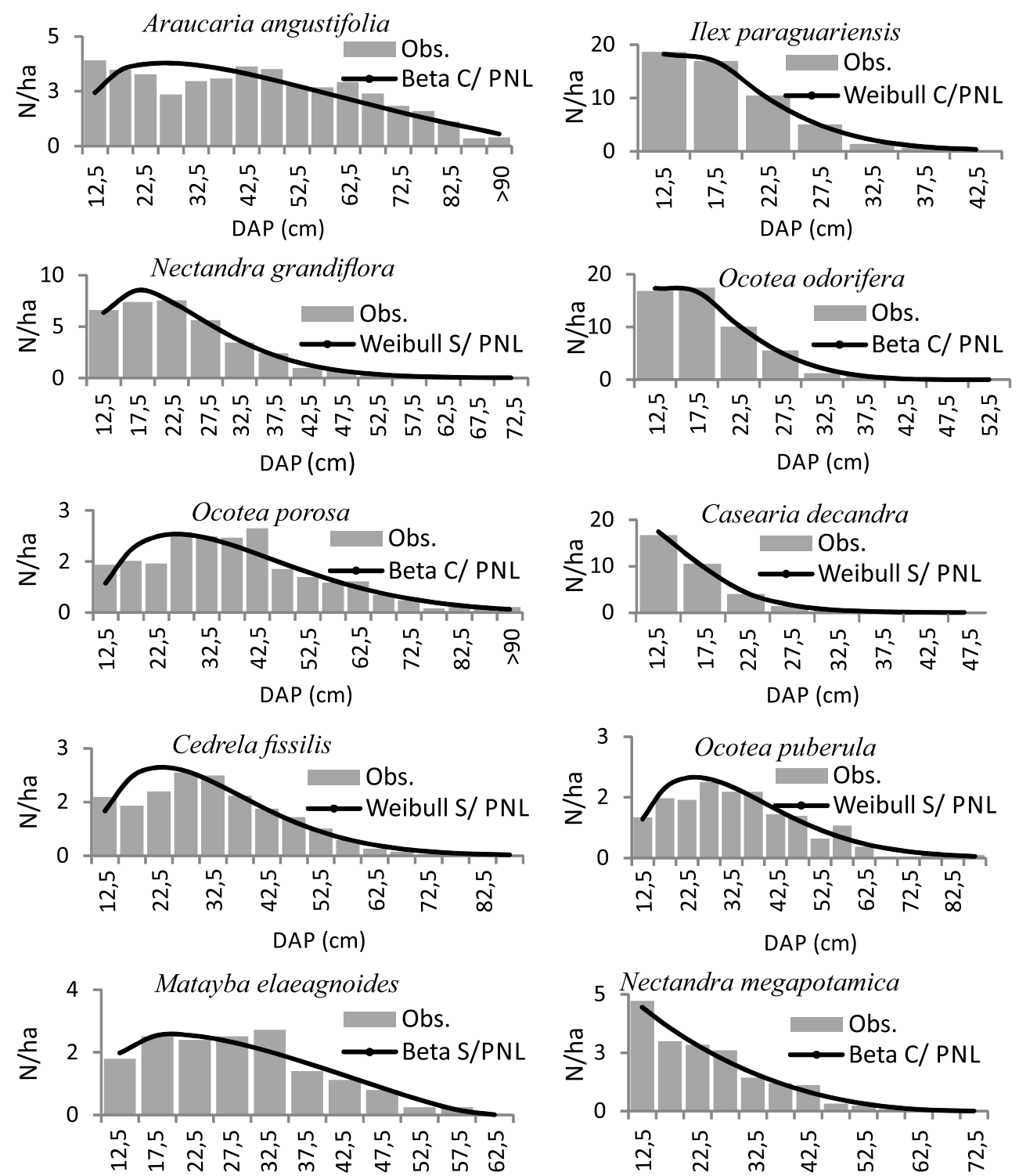
$\operatorname{DAP}(\mathrm{cm})$
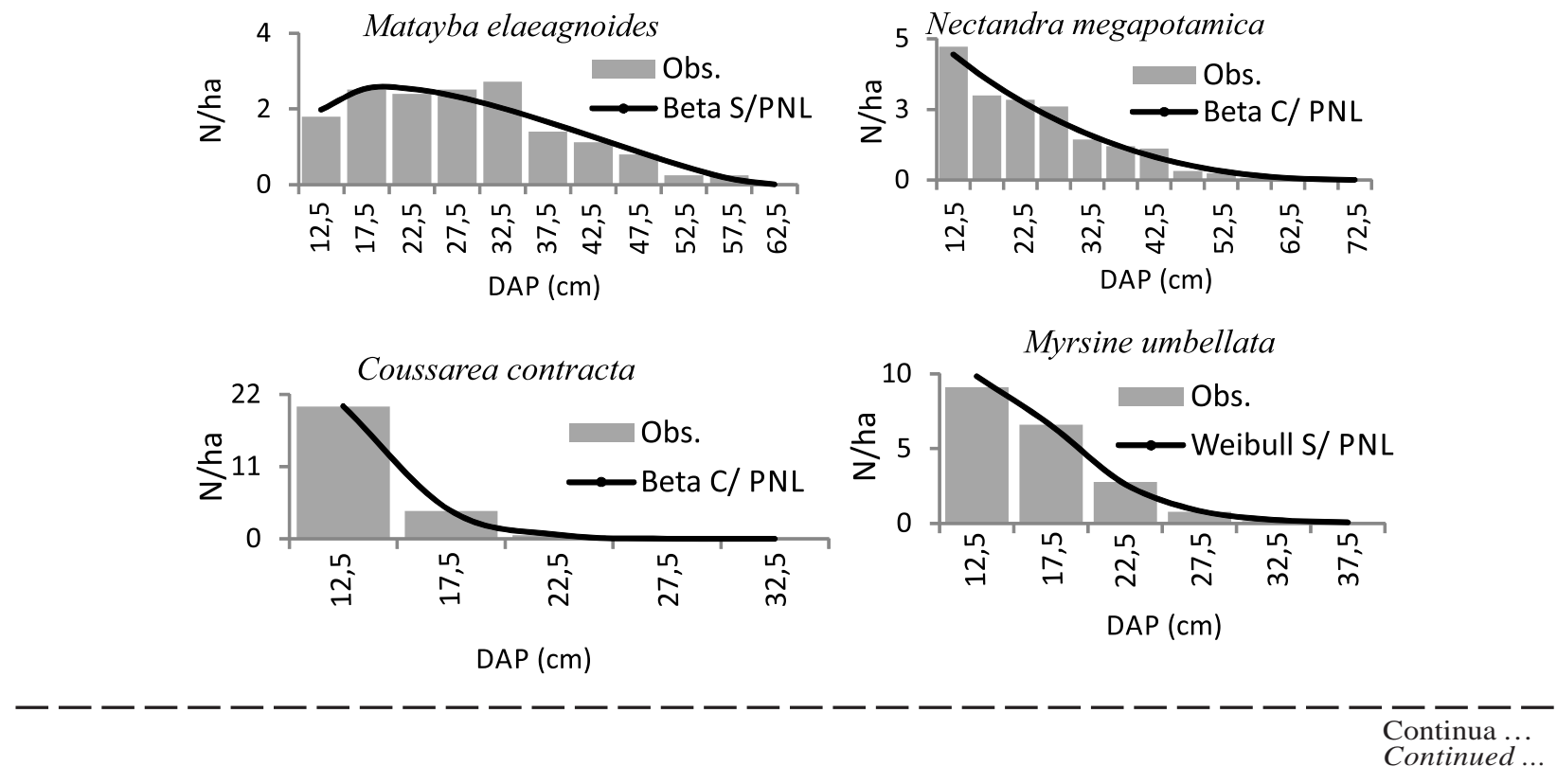

Revista Árvore, Viçosa-MG, v.38, n.2, p.297-308, 2014 
Figura 1 - Cont.

Figure 1-Cont.

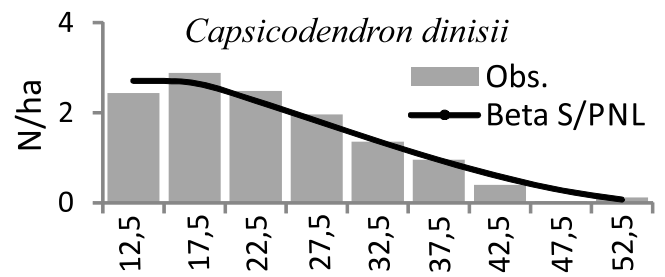

$\operatorname{DAP}(\mathrm{cm})$
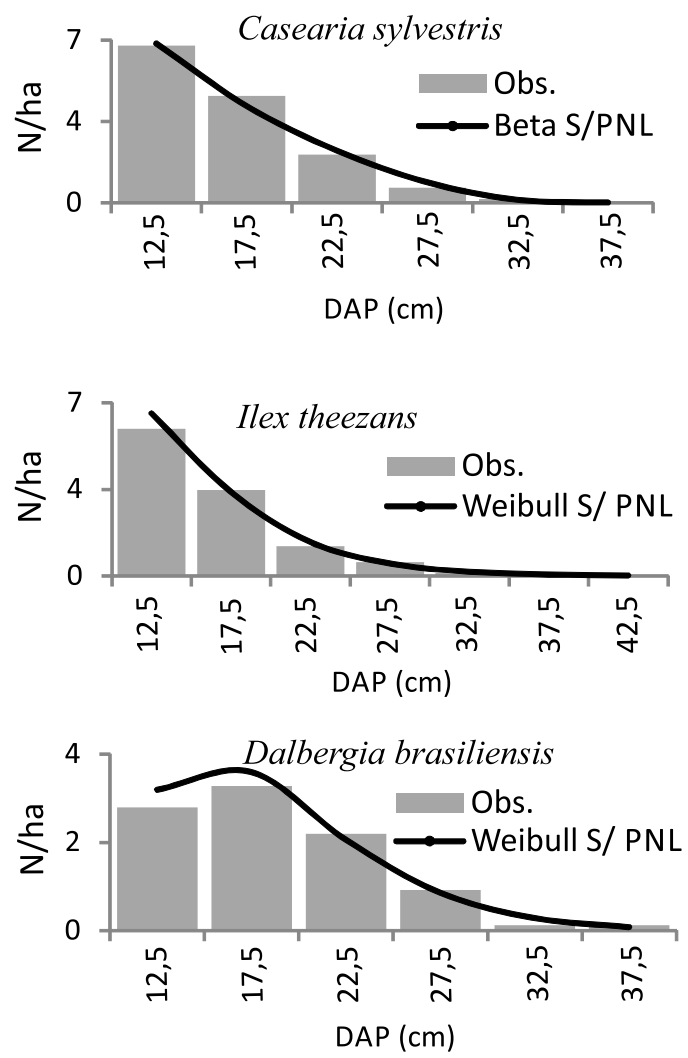

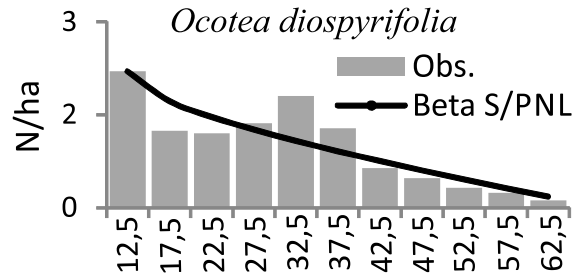

$\operatorname{DAP}(\mathrm{cm})$
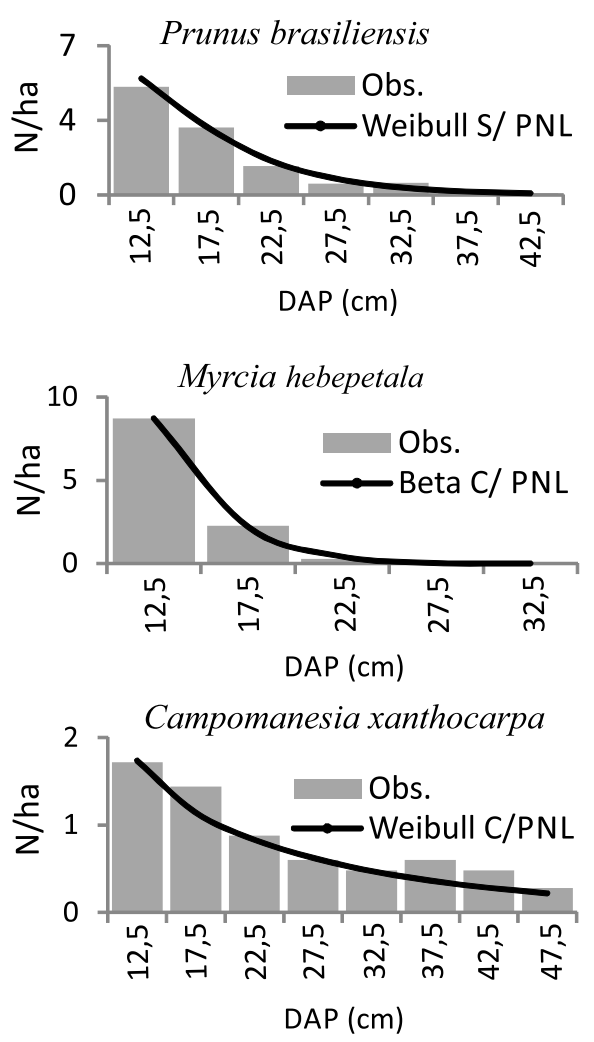

Figura 1 - Número de árvores por hectare (N/ha) observado e estimado pelas funções de melhor desempenho das 20 espécies de maior valor de importância (VI).

Figure 1 - Number of trees per hectare (N/ha) observed and predicted by the functions with best performances for the 20 species with the highest importance values (VI).

comercial e algumas mais frequentes para as amplitudes diamétricas de 10, 7 e 5 cm. O diâmetro mínimo utilizado foi de $15 \mathrm{~cm}$. Concluiu que a Exponencial, a Polinomial de Goff \& West e a Beta foram as que melhor se ajustaram, e a de melhor amplitude para os dados foi de $10 \mathrm{~cm}$.
Campos e Turnbull (1981) afirmaram que a função Beta, embora tenha proporcionado ótimo ajuste em seus estudos de prognose da produção florestal, apresenta desvantagem quando comparada com a função Weibull, devido ao fato de a primeira não ser facilmente relacionada a certos atributos do povoamento.

Revista Árvore, Viçosa-MG, v.38, n.2, p.297-308, 2014 

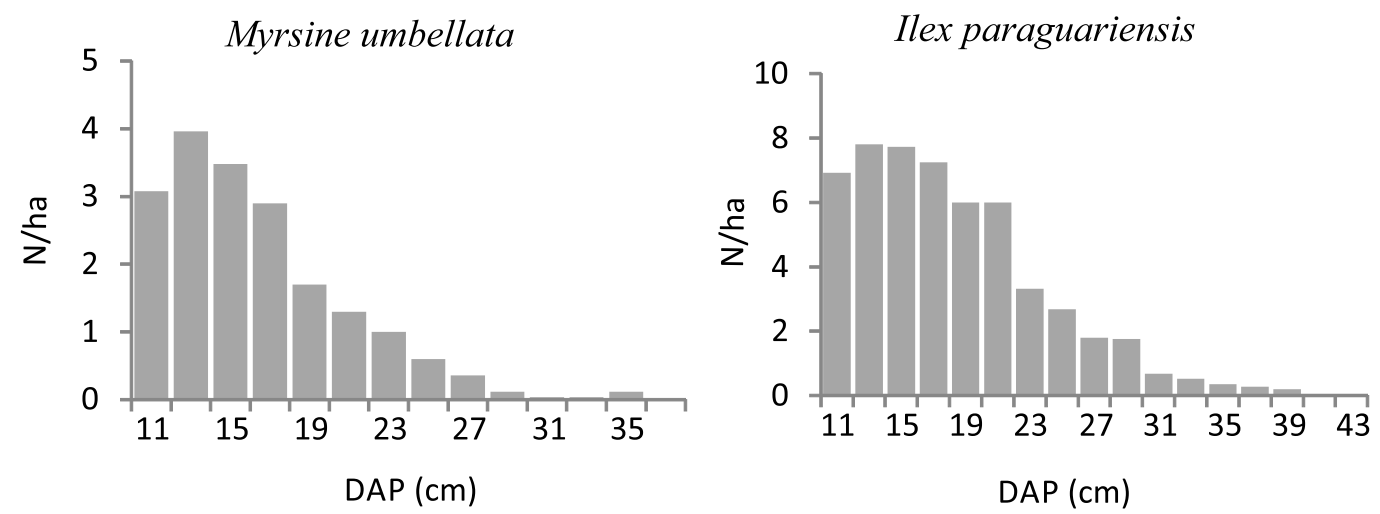

Figura 2 - Distribuição diamétrica da Myrsine umbellata e Ilex paraguariensis indicando a tendência da unimodalidade com assimetria positiva dos dados agrupados em intervalos de $2 \mathrm{~cm}$.

Figure 2 - Diameter distribution of Myrsine umbellata and Ilex paraguariensis indicating the tendency of unimodality with positive skewness for $\mathrm{DBH}$ data grouped in $2 \mathrm{~cm}$ width classes.

Quanto à forma das distribuições diamétricas, verifica-se nas Figuras 1 que a maioria das espécies apresentou grande número de árvores nas primeiras classes, caracterizando boa regeneração e continuidade na perpetuação da espécie. Algumas espécies se diferenciaram pelo maior número de indivíduos na primeira classe, definindo a distribuição na forma de “J-invertido". Outras espécies apresentaram a moda não na primeira classe, mas próxima a ela, nas classes subsequentes, caracterizando a distribuição como unimodal com assimetria positiva.

A distribuição irregular de espécies como a Araucaria angustifolia e algumas espécies da família das Lauráceas, conhecidas pelo grande valor comercial de sua madeira, sugere a ação antrópica com cortes seletivos em alguma época passada.

Entre as espécies analisadas, verificou-se a presença de árvores em quase todas as classes, porém em menor número nas maiores classes diamétricas, seguindo a dinâmica natural da floresta, em que poucos indivíduos atingem grandes dimensões, devido à intensa disputa por luz, água e nutrientes com outras espécies ao longo do seu ciclo de vida.

Silva et al. (2004) afirmaram que a permanência de determinada espécie em uma comunidade natural depende, entre outros fatores, da existência de distribuição regular do número de indivíduos por classes de diâmetro, de tal modo que possa garantir sua estabilidade futura, assim como da ocorrência de árvores reprodutivas. Acrescentaram que, desse modo, baixas densidades populacionais de adultos e regenerantes são indicativos de que a espécie poderá vir a ser substituída durante a evolução da floresta, e a sua efetiva conservação in situ só poderá ocorrer se todas as fases de crescimento forem mantidas no ambiente onde a espécie se desenvolve.

Schaaf et al. (2006) afirmaram que se uma espécie tem poucos indivíduos nas classes inferiores, mas pequena probabilidade de morrer devido à competição, é bem provável que tal espécie se mantenha na floresta. No entanto, esses autores acrescentaram que uma espécie na qual grande parte dos indivíduos esteja sujeita a morrer devido à competição necessita apresentar grande frequência nas classes diamétricas inferiores para ter alguma chance de sobreviver na comunidade.

Carvalho (1981), estudando algumas espécies da Amazônia, determinou que espécies heliófilas como a cupiúba (Goupia glabra) não apresentaram indivíduos nas primeiras classes em decorrência de a floresta oferecer pouca penetração de luz. Outras espécies estudadas, como a andiroba (Carapa guianensis), jarana (Holopyxidium jarana) e feijó-branco (Cordia bicolor), apresentaram distribuições decrescentes.

De modo geral, foram encontradas grandes diferenças nas formas de distribuições diamétricas, bem como na frequência do número de árvores nas classes diamétricas, inclusive entre espécies da mesma família, como é o caso das Lauráceas, representadas neste trabalho pelas canelas, além da imbuia, que apresentaram grande amplitude na frequência de árvores entre as classes. Enquanto a frequência nas primeiras classes da canela-sassafrás é de aproximadamente 15- 
20 árvores/ha, em outras, como a canela-guaicá, a canela-pimenta e a imbuia, é de 1-2 árvores/ha. Frequências intermediárias foram encontradas na canelaimbuia e na canela-amarela (aproximadamente de 56 árvores, em média, nas primeiras classes). Canalez et al. (2006) também encontraram grandes diferenças no número de árvores nas classes diamétricas de espécies de Lauráceas. Na canela-amarela, esses autores encontraram frequência próxima a 40 árvores/ha na primeira classe. Outras espécies como a canela-guaicá, canela-imbuia e imbuia não passaram de cinco árvores nessa classe.

Verificou-se que a forma das distribuições diamétricas das espécies são influenciadas pelas suas características ecológicas. Além disso, o intervalo de classe utilizado para agrupar os dados de diâmetro interfere diretamente na forma da distribuição. Outro fator importante a ser considerado é que uma espécie ao ser submetida a qualquer intervenção, dependendo do regime de manejo em que ela seja submetida, poderá descaracterizar sua estrutura diamétrica e, consequentemente, sua distribuição.

\section{CONCLUSÃO}

- Das 20 espécies analisadas, 13 apresentaram a distribuição diamétrica com tendência decrescente e sete exibiram distribuição com tendência unimodal com assimetria positiva, para dados agrupados em classes de $5 \mathrm{~cm}$ de DAP.

- Nas análises dos ajustes das 20 espécies de maior IVI, o índice de Reynolds indicou como mais adequada a distribuição Weibull 3P para 10 espécies e a Beta para as outras 10. Resultados similares foram também indicados pelo Erro-Padrão da Estimativa.

- A utilização da Programação Não Linear (PNL) nem sempre melhorou os ajustes conforme se esperava. Entre as 20 espécies analisadas, houve redução do desvio entre distribuição observada e estimada com o uso dessa ferramenta de otimização, em oito casos. No entanto, recomenda-se a aplicação desse procedimento na tentativa de obter melhores ajustes de funções densidade de probabilidade para descrever distribuições diamétricas.

- A definição do intervalo de classe influencia, sobremaneira, a forma da distribuição diamétrica.

\section{REFERÊNCIAS}

BAILEY, T. R. L.; DELL, R. Quantifying diameter distributions with the Weibull function. Forest Science, v.19, n.2, p.97-104, 1973.

BARRA, O. S. V. et al. Proposta metodológica para ajuste ótimo da distribuição diamétrica Weibull 3P. Revista Floresta, v.34, n.3, p.387393, 2004.

\section{BARROS, P. L. C. Estudo das distribuições} diamétricas da floresta do Planalto Tapajós - Pará. 123f. Dissertação (Mestrado em Ciências Florestais) - Universidade Federal do Paraná, Curitiba, 1980.

BUNYAVEJCHEWIN, S. et al. Stand structure of a seasonal dry evergreen forest at Huai Kha Hhaeng Wildlife Sanctuary, western Thailand. Natural History. Bulletin of the Siam Society, n.50, p.125-134, 2001.

CAMPOS, J. C. C.; TURNBULL, K. J. Um sistema para estimar produção por classe de diâmetro e sua aplicação na interpretação de efeitos de desbaste. Revista Árvore, v.5, n.1, p.1-16, 1981.

CANALEZ, G. G.; CORTE, A. P. D.; SANQUETTA, C. R. Dinâmica da estrutura da comunidade de Lauráceas no período 1995-2004 em uma floresta de araucária no sul do estado do Paraná, Brasil. Ciência Florestal, v.16, n.4, p.357-367, 2006.

CAO, Q. V. Predicting parameters of a weibull function for modeling diameter distribution. Forest Science, v.50, n.5, p.682-685, 2004.

CARVALHO, J. O. P. Distribuição diamétrica de espécies comerciais e potenciais em floresta tropical úmida natural na Amazônia. Belém, EMBRAPA CPATU, 1981. 34p. (Boletim de Pesquisa, 23)

DISPERATI, A. A. et al. Aplicação da técnica “filtragem de locais máximas” em fotografia aérea digital para a contagem de copas em reflorestamento de Pinus elliottii. Revista Scientia Forestalis, n.76, p.45-55, 2007.

DODSON, B. The weibull analysis

handbook. 2.ed. New York: Quality Press, 2006. 162p.

Revista Árvore, Viçosa-MG, v.38, n.2, p.297-308, 2014 
FINGER, C. A. G. - Fundamentos da biometria florestal. Santa Maria: UFSM/ CEPEF/FATEC, 1992. 269p.

INSTITUTO BRASILEIRO DO MEIO AMBIENTE E DOS RECURSOS NATURAIS RENOVÁVEIS IBAMA. Disponível em < $\underline{w w w . i b a m a . g o v . b r}>$ Acesso em 10 de nov. de 2009.

LOEWENSTEIN, E. F.; JOHNSON, P. S.; GARRETT, H. E. Age and diameter structure of a managed uneven-aged oak forest. Canadian Journal of Forest Research, v.30, n.7, p.1060-1070, 2000.

MACHADO, S. A.; BARTOSZEK, A. C. P. S.; OLIVEIRA, E. B. Estudo da estrutura diamétrica para Araucaria angustifolia em florestas naturais nos estados da região sul do Brasil. Revista Floresta, v.26, n.1/2, p.59-70, 1997.

PEUHKURINEN, J.; MALTAMO, M.; MALINEN, J. Estimating species-specific diameter distributions and saw log recoveries of boreal forests form airbone laser scanning data and aerial photographs: a distribution-based approach. Silva Fennica, v.42, n.4, p.625-641, 2008.

PODLASKI, R. Suitability of the selected statistical distributions for fitting diameter data in distinguished development stages and phases of near-natural mixed forest in the Swietokrzyski National Park (Poland). Forest Ecology and Management, v.236, n.2, p.393-402, 2006.

QIN, J.; CAO, Q. V.; BLOUIN, D. C. Projection of a diameter distribution through time. Canadian Journal of Forest Research, v.37, n.1, p.188-194, 2007.

REYNOLDS JR., M. R.; BURK, T. E.; HUANG, W. C. Goodness-of-fit tests and model selection procedures for diameter distribution models. Forest Science, v.34, n.2, p.373-399, 1988.
SAWCZUK, A. R. Alteração na florística e estrutura horizontal de um fragmento de floresta ombrófila mista na Floresta Nacional de Irati, estado do Paraná. 2009. 147f. Dissertação (Mestrado em Ciências Florestais) - Universidade Estadual do Centro-Oeste, Irati, 2009.

SCHAAF, L. B. et al. Alteração na estrutura diamétrica de uma floresta ombrófila mista no período entre 1979 e 2000. Revista Árvore, v.30, n.2, p.283-295, 2006.

SCOLFORO, J. R. S. Modelagem do crescimento e da produção de florestas plantadas e nativas. Lavras: UFLA/FAEPE, 1998. 441p.

SIIPILEHTO, J. Modeling stand structure in young Scots pine dominated stands. Forest Ecology and Management, v.257, p.223232, 2009.

SILVA, J. A. et al. Distribuição diamétrica de indivíduos de Aspidospema spp. na Reserva Genética Tamanduá, DF. In: SIMPÓSIO LATINO-AMERICANO SOBRE MANEJO FLORESTAL, 3., 2004, Santa Maria. Anais... Santa Maria: Universidade Federal de Santa Maria, 2004.

TORRES-ROJO, J. M.; TORRES MARGAÑA, O. S.; MIRELES, M. A. Metodologia para mejorar la predicción de parametros de distribuciones diametricas. Revista Agrociencia, v.34, n.5, p.627-637, 2000.

WESTPHAL, C. et al. Is the reverse J-shaped diameter distribution universally applicable in European virgin beech forests? Forest Ecology and Management, v.223, n.1, p.75-83, 2006. 